\title{
Alpha-Fetoprotein as a Predictive Marker for Patients with Hepatitis B-Related Acute-on-Chronic Liver Failure
}

\author{
Xiaoping Wang, ${ }^{1,2}$ Caifei Shen, ${ }^{1}$ Jianjiang Yang, ${ }^{1}$ Xianjun Yang, ${ }^{3}$ Sen Qin, ${ }^{1,2}$ \\ Haijun Zeng, ${ }^{1}$ Xiaoling $W u,{ }^{1}$ Shanhong Tang $\mathbb{i},{ }^{1,2}$ and Weizheng Zeng ${ }^{1}{ }^{1}$ \\ ${ }^{1}$ Department of Gastroenterology, Chengdu Military General Hospital, Chengdu, Sichuan, China \\ ${ }^{2}$ College of Medicine, Southwest Jiaotong University, Chengdu, Sichuan, China \\ ${ }^{3}$ Chengdu Military Command Disease Prevention and Control Center, Chengdu, Sichuan, China \\ Correspondence should be addressed to Shanhong Tang; 15928956390@163.com and Weizheng Zeng; zengweizheng@163.com
}

Received 3 February 2018; Revised 29 March 2018; Accepted 12 April 2018; Published 9 May 2018

Academic Editor: Yu-Chen Fan

Copyright (C) 2018 Xiaoping Wang et al. This is an open access article distributed under the Creative Commons Attribution License, which permits unrestricted use, distribution, and reproduction in any medium, provided the original work is properly cited.

\begin{abstract}
Background and Aims. The value of alpha-fetoprotein (AFP) in hepatitis B-related acute-on-chronic liver failure (HBACLF) is not fully understood. The present study aimed to evaluate the prognostic effect of AFP on the prediction of HBACLF outcomes. Methods. We investigated a cohort of patients with HBACLF admitted from January 2013 to May 2017. The endpoint of followup was 180 days, death, or liver transplantation. AFP concentrations were estimated on admission. To make statistical comparisons, we used chisquared test, receiver operating characteristic (ROC) curve analysis, survivorship curve analysis, and Cox proportional-hazards model. Results. A total of 92 patients ( $81.5 \%$ male, median age of 46 years) were included. Overall survival rate within 180 days was $43.48 \%$, and the value of $\log _{10}{ }^{A F P} \geq 2.04$ indicated a better prognosis with $76.9 \%$ specificity and $62.5 \%$ sensitivity for patients with HBACLF. Age (HR 1.041), total bilirubin (HR 1.004), $\log _{10}$ AFP (HR 2.155), and INR (HR 1.446) were found to be risk factors of survival. Conclusion. AFP could be a useful marker to predict outcomes of acute-on-chronic liver failure.
\end{abstract}

\section{Introduction}

Acute-on-chronic liver failure (ACLF) is defined by a rapid progression in hepatic dysfunction induced by certain precipitating events due to previous liver diseases, resulting in multisystem organ failure and high short-term mortality [1]. There is no specific treatment for ACLF, and the most effective therapy is liver transplantation. However, the shortage of liver donations has largely hindered its wide implementation. Supporting the regeneration of hepatocytes and preventing the complications tend to decrease the mortality of ACLF, and artificial liver support is a useful method to manage ACLF [2]. The aetiologies of ACLF vary between territories. Viral infections are more common in Asia, while there is a wide prevalence of alcoholic cirrhosis and nonalcoholic fatty liver in American and European countries [3-6]. With a carrier rate of hepatitis B virus (HBV) surface antigen at approximately $8 \%$ in adults, China exhibits a high morbidity of hepatitis $\mathrm{B}$, which is the most common aetiological factor of ACLF [7].
Some scoring systems efficiently assess the severity and mortality of ACLF, such as SOFA score [8], MELD [9], and King's College Criteria [10]. Other parameters including bilirubin and international normalized ratio (INR) are treated as prognostic markers in clinical practice as well. Those methods primarily focus on the impaired hepatic function, while seldom did researchers concentrate on parameters of hepatocyte regeneration and evaluate their prognostic value for ACLF. Typically, AFP is the most abundant plasma protein in foetuses, and serum AFP remains elevated in infant livers until several weeks after birth. High serum AFP expression in adults generally indicates a high possibility of hepatocellular carcinoma in patients with chronic hepatitis or cirrhosis [11]. Meanwhile, AFP is considered a biomarker of proliferating liver stem cells in liver injury conditions as well, and the recruitment of liver progenitor cells is associated with a better outcome for liver failure [12, 13].

The discovery of AFP dates back to 1960s. Since then, AFP has been investigated in the field of liver diseases. Previous 


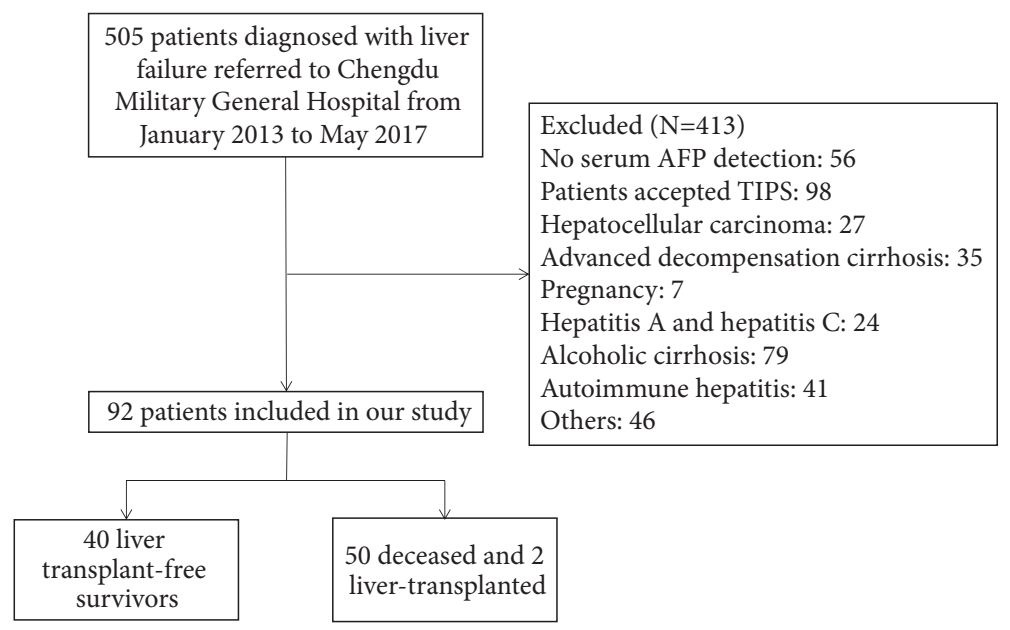

FIgURE 1: Inclusion and exclusion criteria for the study.

studies demonstrated that AFP was a vital prognostic marker for outcomes in patients with acute liver failure [14-16]. However, few studies evaluated the outcomes of ACLF from the perspective of hepatocyte regeneration. As we know, ACLF exhibits a small window during which liver dysfunction may be reversed, and the repair of liver tissues is tightly correlated with hepatocyte regeneration, which presents as increased AFP levels. Therefore, we performed a retrospective study to identify whether AFP was a valid predictive indicator of outcomes in ACLF patients.

\section{Materials and Methods}

2.1. Study Population. We concentrated on a cohort of patients with hepatitis B-related acute-on-chronic liver failure (HBACLF). A total of 505 patients with suspected ACLF were enrolled in our study from January 2013 to May 2017 at Chengdu Military General Hospital, Sichuan, China. Researchers who undertook the work of selecting cases as the main subjects were blind to the serum AFP concentrations of those patients. ACLF is diagnosed according to the criteria of Asian Pacific Association for the Study of the Liver (APASL): serum bilirubin $\geq 85 \mu \mathrm{mol} / \mathrm{L}$, INR $\geq 1.5$ or prothrombin activity $\leq 40 \%$, any degree of encephalopathy and/or clinical ascites within 4 weeks, and an evidence of ongoing chronic liver diseases [3]. Patients who were diagnosed with ACLF and aged 18 to 75 years were included. A total of 413 patients in our database were excluded for the following reasons: (1) lack of serum AFP concentrations; (2) manifestation of decompensated liver cirrhosis prior to ACLF diagnosis, such as ascites and variceal haemorrhage; (3) patients with portal hypertension who received a transjugular intrahepatic portosystemic shunt (TIPS); (4) patients pathologically diagnosed with or clinically suspected for hepatocellular carcinoma; (5) other malignancies such as gastric cancer; (6) pregnancy; (7) HIV or hepatotropic virus infection other than HBV; and (8) other preexisting chronic liver diseases, such as fatty liver, autoimmune hepatitis, and alcoholic cirrhosis (Figure 1). The final cohort contained 92 patients with a median age of 46 years (range, 18-75 years), and all patients received antiviral therapy by orally taking tenofovir or entecavir. Besides, reduced glutathione was given to protect the liver from subsequent damage.

2.2. Clinical and Biological Parameters. The clinical parameters included ascites and hepatic encephalopathy (HE). The biological parameters included AFP, INR, and total bilirubin. The serum AFP levels were measured on admission.

2.3. Followup. The end point of observation was 180 days, death, or liver transplantation. Forty of the 92 patients survived spontaneously, 50 patients died, and 2 patients received liver transplantation.

2.4. Statistical Analysis. Results are presented as means and standard deviations (SDs) and median and range appropriately. The chi-squared test was used to compare rates between groups. Receiver operating characteristic (ROC) curve analysis was performed. Survival was estimated by Kaplan-Meier method, and differences were evaluated with log-rank test. Cox proportional-hazards model was adopted to estimate the risk factors of survival. Data were analyzed using SPSS version 16.0 software (IBM Corporation, Somers, NY, USA). Differences were considered to be of statistical significance when the $P$ value $\leq 0.05$.

\section{Results}

3.1. Baseline Characteristics. Ninety-two patients were incorporated in our study, including 17 women (18.5\%). The population was divided into two groups based on the prognosis of ACLF. In total, there were 40 liver transplant-free survivors, 50 deceased patients, and 2 liver-transplanted patients. Table 1 depicts the demographic and biochemical characters of the two groups. Age, total bilirubin, AFP, and INR differed significantly between transplant-free survivors and those who deceased or got liver-transplanted.

3.2. AFP as a Predictor for Prognosis of HBACLF. The recruitment of functional hepatocytes is the key to the recovery 
TABLE 1: Characteristic comparisons between subgroups.

\begin{tabular}{lccc}
\hline Variables & $\begin{array}{c}\text { Transplant-free survival } \\
\text { group }(n=40)\end{array}$ & $\begin{array}{c}\text { Deceased and transplanted } \\
\text { group }(n=52)\end{array}$ & $P$ value \\
\hline Age $^{*}, \mathrm{y}$ & $41.83(12.37)$ & $50.31(13.10)$ & 0.002 \\
Male $n, \%$ & $33(82.50 \%)$ & $42(80.77 \%)$ & 0.832 \\
Total bilirubin ${ }^{\#}, \mu \mathrm{mol} / \mathrm{L}$ & $261.89(86.98-496.59)$ & $386.55(137.45-723.22)$ & $<0.001$ \\
$\mathrm{AFP}^{\#}, \mathrm{ng} / \mathrm{ml}$ & $148.80(8.50-2375.30)$ & $43.21(1.10-1495.80)$ & $<0.001$ \\
INR $^{\#}$ & $1.83(1.50-4.31)$ & $2.21(1.51-5.70)$ & 0.001 \\
\hline
\end{tabular}

AFP, alpha-fetoprotein; INR, international normalized ratio; ${ }^{*}$ normally distributed continuous variable; ${ }^{\#}$ not normally distributed continuous variable.

TABLE 2: Univariate and multivariate Cox regression analysis for survival.

\begin{tabular}{|c|c|c|c|c|c|c|c|c|}
\hline \multirow{3}{*}{$\begin{array}{l}\text { Parameter } \\
\text { Age }^{*}\end{array}$} & \multicolumn{4}{|c|}{ Univariate Cox regression $(n=92)$} & \multicolumn{4}{|c|}{ Multivariate Cox regression $(n=92)$} \\
\hline & \multirow{2}{*}{$\begin{array}{c}P \\
0.003\end{array}$} & \multirow{2}{*}{$\frac{\text { HR }}{1.033}$} & \multicolumn{2}{|c|}{$\mathrm{CI}$} & \multirow{2}{*}{$\begin{array}{c}P \\
<0.001\end{array}$} & \multirow{2}{*}{$\begin{array}{c}\text { HR } \\
1.041\end{array}$} & \multicolumn{2}{|c|}{$\mathrm{CI}$} \\
\hline & & & 1.011 & 1.056 & & & 1.019 & 1.063 \\
\hline Total bilirubin* & $<0.001$ & 1.004 & 1.002 & 1.006 & $<0.001$ & 1.004 & 1.002 & 1.006 \\
\hline $\mathrm{INR}^{*}$ & 0.001 & 1.564 & 1.200 & 2.039 & 0.015 & 1.446 & 1.074 & 1.948 \\
\hline $\log _{10}{ }^{A F P \#}$ & $<0.001$ & 2.908 & 1.603 & 5.274 & 0.018 & 2.155 & 1.139 & 4.076 \\
\hline Ascites ${ }^{\#}$ & 0.407 & & & & & & & \\
\hline $\mathrm{HE}^{\#}$ & 0.163 & & & & & & & \\
\hline
\end{tabular}

AFP, alpha-fetoprotein; INR, international normalized ratio; HE, hepatic encephalopathy; HR, hazard ratio; and CI, confidence interval; ${ }^{*}$ continuous variables and ${ }^{\#}$ categorical variables $\left(\log _{10}{ }^{\text {AFP }}\right.$ was sorted into subgroups: $\log _{10}{ }^{\text {AFP }} \geq 2$ and $\log _{10}{ }^{\text {AFP }}<2$. No statistical significance was attained between patients with or without ascites and $\mathrm{HE})$.

of the impaired liver function. To estimate the outcome of ACLF from the perspective of hepatocyte regeneration, we evaluated the predictive value of AFP by creating an equation, namely, $\log _{10}$ AFP , to assess the prognosis of HBACLF. A receiver operating characteristic curve was created for this parameter to predict the outcome of ACLF patients. The area under the curve was 0.725 . A cut-off point of $\log _{10}{ }^{A F P} \geq 2.04$ was suggested to indicate a better outcome with $76.9 \%$ specificity and $62.5 \%$ sensitivity (Figure 2).

3.3. $\log _{10}$ AFP Is a Risk Factor of Survival for HBACLF. Patients with chronic hepatitis B-related ACLF exhibited an incredibly high mortality within 180 days in our observation. The transplant-free survival rate at 30 days was $72.83 \%$, and it gradually declined to $43.48 \%$ at 180 days of followup (Figure 3(a)). Given that patients would probably have a better outcome when $\log _{10}$ AFP was approximately higher than 2, we then chose $\log _{10}$ AFP as a categorical variable to assess the survival of HBACLF. In addition, we also adopt demographic parameters and serum biochemical parameters which were considered as representatives of the hepatic function. By using Cox proportional-hazards model, $\log _{10}$ AFP was found to be an independent factor of survival as a categorical variable, as with continuous variables including age, INR, and levels of total bilirubin (Table 2). There were 41 patients whose $\log _{10}$ AFP $\geq 2$, and this group of patients was more likely to exhibit a longer survival time. The transplant-free survival rates at 30,90 , and 180 days were $58.82 \%$ versus $90.24 \%(P=$ $0.001), 39.22 \%$ versus $83.93 \%(P<0.001)$, and $29.41 \%$ versus

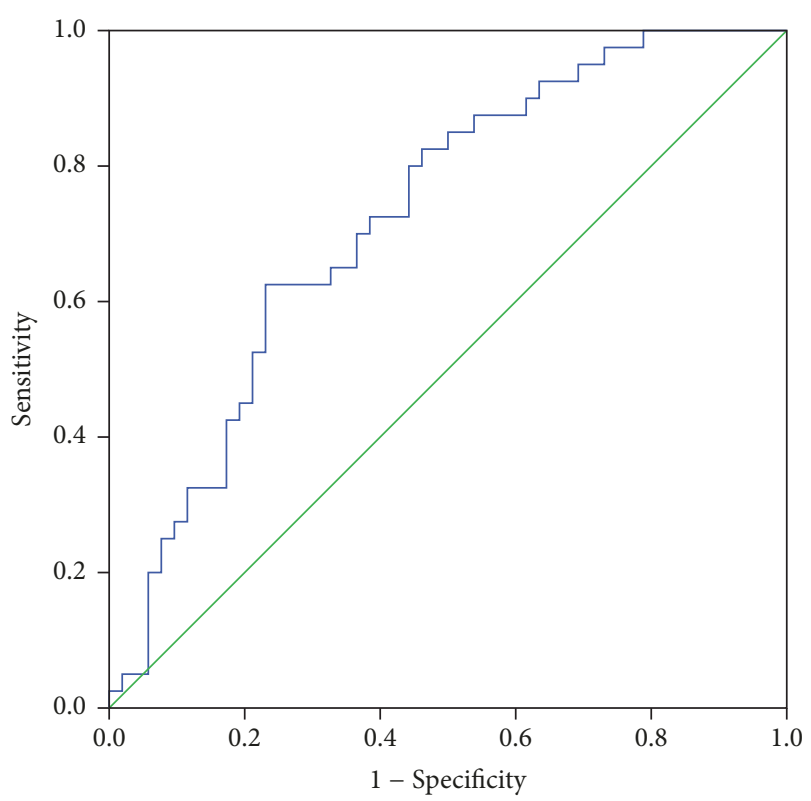

FIGURE 2: ROC curve for $\log _{10}$ AFP in predicting the outcome of $\operatorname{HBACLF}(n=92)$.

58.54\% $(P=0.005)$, respectively, in groups of patients with $\log _{10}$ AFP $<2$ and $\geq 2$ (Figure 3(b)).

\section{Discussion}

The idea of ACLF was first proposed to describe the acute liver damage of an ongoing chronic liver disease in 1995 [17]. There 


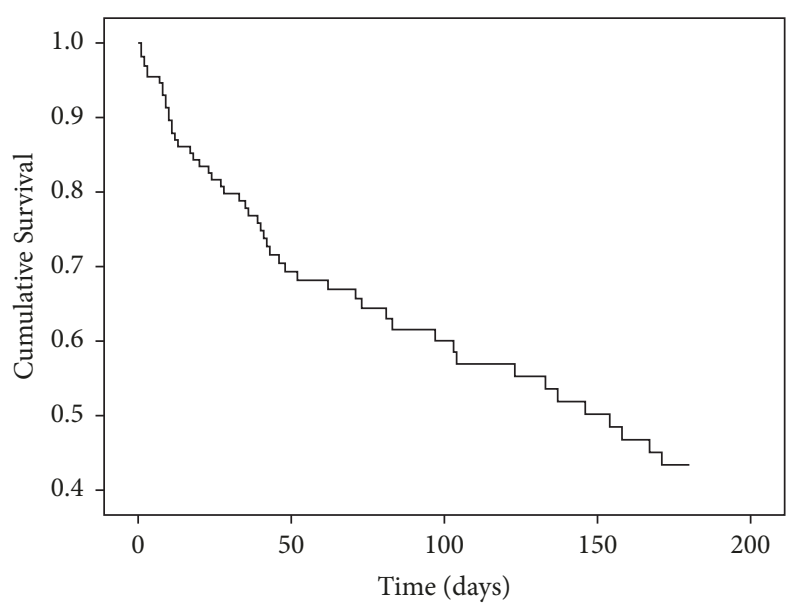

(a)

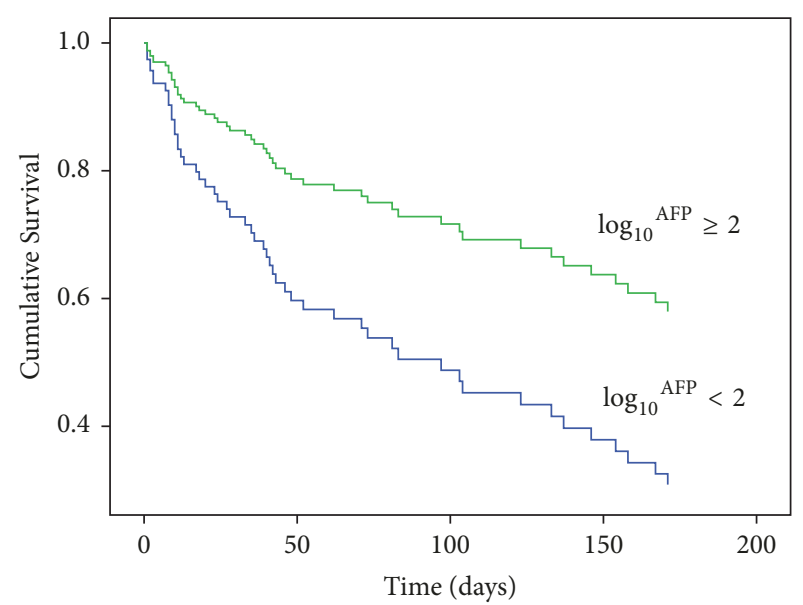

(b)

FIGURE 3: (a) Graph of multivariate Cox regression survival curve of the total population $(n=92)$. (b) Graph of multivariate Cox regression survival curve for patients with $\log _{10}$ AFP $\geq 2(n=41)$ and $\log _{10}$ AFP $<2(n=51)$.

are more than 13 different definitions of ACLF worldwide, which vary from the West to the East. An acute insult may lead to rapid and progressive liver failure in patients with chronic liver diseases and result in high short-term mortality of approximately $50-90 \%$ because of the limited functional reserve of the liver $[1,18]$.

China exhibits a high morbidity of hepatitis B and HBACLF. There are studies estimating the severity and outcomes of HBACLF by establishing prognostic scoring models. Previous studies illustrated that the liver volume [19], lymphocytemonocyte ratio [20], albumin-bilirubin score [21], logistic regression model [22], macrophage inflammatory protein $3 \alpha$ [23], and other methods [24-26] could be simple and sensitive models to evaluate the severity and prognosis of ACLF, which would be practically useful in clinic. Those methods mainly concentrated on the severity of liver injury, and the parameters in those models were generally to reflect the condition of liver function. It is known to us that the prognosis of ACLF depends on the extent of liver injury, the capability of hepatocyte regeneration, and the prevention of multiple organ failure. There are a number of studies evaluating the prognosis of ACLF from the perspective of liver function; however, limited researches have assessed the outcome of ACLF by adopting parameters reflecting hepatocyte regeneration. AFP is a biomarker of liver renovation; thus, we investigated the prognostic value of AFP in ACLF for that the magnitude of in the increased AFP levels is closely related to hepatocyte regeneration after acute or superimposed hepatic injury. Previous studies demonstrated that AFP was a prognostic marker in patients with acute liver failure [27, 28]. However, limited studies demonstrated a correlation between AFP levels and the outcomes of patients with ACLF, especially patients with acute hepatic failure based on chronic HBV infection.

AFP is not detected in normal adult serum. In 1963, Abelev et al. [29] found that a type of murine embryonal $\alpha$ globulin, which was originally called AFP, could be detected in normal or malignant hepatocyte proliferation in adult rats. Then, AFP was considered a marker of hepatocyte regeneration, and the capacity of hepatocyte regeneration is key to the reversal of liver injury. The present study assessed the predictive value of AFP in the prognosis of hepatitis Brelated acute-on-chronic liver failure because these patients always exhibited bad progress, and the short-term mortality is dramatically high. Valid predictive models and intensive care are necessary in the management of ACLF because some individuals regain their health following liver injury recovery and hepatocyte regeneration. Our study demonstrated that the parameter $\log _{10}$ AFP was a helpful marker to predict the outcomes of ACLF. A higher AFP concentration could predict a better outcome of HBACLF, and $\log _{10}$ AFP $\geq 2$ could indicate a longer short-term survival time for patients with ACLF. Therefore, we speculated that the concentration of AFP may be positively related to the capacity for liver regeneration in the condition of acute liver injury on the basis of chronic liver diseases, apart from malignancies such as hepatocellular carcinoma and gastric cancer.

Consistent with Katoonizadeh's research [18], our study indicated that the difference of age was of statistical significance between survivors and those who deceased or got liver transplanted. Elderly patients exhibit a weakened condition of body function, which may result in multiple organ failure when there was a hypohepatia already. And we suspected that the number of functional hepatocytes may be lower in the elderly than young adults. In addition, total bilirubin and INR were found to be risk factors of survival as well. Previous studies demonstrated that a higher level of bilirubin was associated with a poor outcome in patients with liver failure because the dysfunctional liver exhibited deficient bilirubin metabolism, which demonstrates that the extent of damage to hepatocytes was extremely serious $[8,30]$. Impeded hepatic synthetic function reduces the production of prothrombin and other proteins in the liver, which accounts for the 
higher level of INR, and a higher degree of INR indicates an unfavourable prognosis [31].

One limitation of our study was the lack of dynamic observations of AFP levels. A persistently low AFP may be interpreted as regenerative failure in protracted cases but ultimately leads to death. Some patients with low AFP concentrations on admission ultimately survived because liver tissue repair occurred followed with a serially intensive care. Other parameters, such as $\mathrm{HBeAg}$, creatinine, albumin, and some scoring systems, could also help assess the outcome of ACLF. Therefore, further studies of multifactor-correlated prognostic models are needed.

\section{Conclusion}

In summary, AFP is an indicator of the prognosis of hepatitis B-related acute-on-chronic liver failure. Higher levels of AFP concentrations could predict a better outcome of HBACLF, and $\log _{10} A F P \geq 2$ would indicate a longer survival time.

\section{Data Availability}

All data arising from this study are contained within the manuscript.

\section{Conflicts of Interest}

The authors declare that they have no competing financial interest.

\section{Authors' Contributions}

Shanhong Tang designed the study and carried it out. Sen Qin and Haijun Zeng joined in data collection. Xianjun Yang and Jianjiang Yang conducted data analysis. Xiaoping Wang and Caifei Shen drafted the manuscript. Xiaoling $\mathrm{Wu}$ and Weizheng Zeng helped to finalize the manuscript. All of the authors read and approved the manuscript. Xiaoping Wang and Caifei Shen equally contributed to this work.

\section{Acknowledgments}

This work was supported by the grant from the National Natural Science Foundations of China 81401993 (to Shanhong Tang).

\section{References}

[1] S. K. Sarin and A. Choudhury, "Acute-on-chronic liver failure: Terminology, mechanisms and management," Nature Reviews Gastroenterology \& Hepatology, vol. 13, no. 3, pp. 131-149, 2016.

[2] L. L. Kjaergard, J. Liu, B. Als-Nielsen, and C. Gluud, "Artificial and bioartificial support systems for acute and acuteon-chronic liver failure: A systematic review," Journal of the American Medical Association, vol. 289, no. 2, pp. 217-222, 2003.

[3] S. K. Sarin, C. K. Kedarisetty, Z. Abbas et al., "Acute-onchronic liver failure: consensus recommendations of the Asian Pacific Association for the Study of the Liver (APASL) 2014," Hepatology International, vol. 8, no. 4, pp. 453-471, 2014.
[4] J. Wendon, J. Cordoba, A. Dhawan et al., "EASL clinical practical guidelines on the management of acute (fulminant) liver failure," Journal of Hepatology, vol. 66, no. 5, pp. 1047-1081, 2017.

[5] J. Polson and W. M. Lee, "AAFOL Disease, AASLD position paper: the management of acute liver failure: update 2011," National Guideline Clearinghouse, vol. 65, no. 1, pp. 1433-1441, 2011.

[6] J. Cordoba, M. Ventura-Cots, M. Simón-Talero et al., "Characteristics, risk factors, and mortality of cirrhotic patients hospitalized for hepatic encephalopathy with and without acute-onchronic liver failure (ACLF)," Journal of Hepatology, vol. 60, no. 2, pp. 275-281, 2014

[7] I. Merican, R. Guan, D. Amarapuka et al., "Chronic hepatitis B virus infection in Asian countries," Journal of Gastroenterology and Hepatology, vol. 15, no. 12, pp. 1356-1361, 2000.

[8] S. Sen, R. Williams, and R. Jalan, "The pathophysiological basis of acute-on-chronic liver failure," Journal of Liver, vol. 22, no. 2, pp. 5-13, 2002.

[9] P. S. Kamath and W. R. Kim, "The model for end-stage liver disease (MELD)," Hepatology, vol. 45, no. 3, pp. 797-805, 2007.

[10] J. Aguirre-Valadez, A. Torre, M. Vilatobã et al., "Indications for liver transplant," Revista De Investigacion Clinica, vol. 66, no. 6, pp. 534-546, 2014.

[11] Y.-M. Zhou, J.-M. Yang, B. Li et al., "Clinicopathologic characteristics of intrahepatic cholangiocarcinoma in patients with positive serum a-fetoprotein," World Journal of Gastroenterology, vol. 14, no. 14, pp. 2251-2254, 2008.

[12] K. Kakisaka, K. Kataoka, M. Onodera et al., "Alpha-fetoprotein: A biomarker for the recruitment of progenitor cells in the liver in patients with acute liver injury or failure," Hepatology Research, vol. 45, no. 10, pp. E12-E20, 2015.

[13] G. G. Karvountzis and A. G. Redeker, "Relation of alpha fetoprotein in acute hepatitis to severity and prognosis," Annals of Internal Medicine, vol. 80, no. 2, pp. 156-160, 1974.

[14] S. K. Jain, A. Rohatgi, K. K. Raman, and V. K. Sharma, "Study of serum prealbumin and serum alpha fetoprotein in cases of fulminant hepatic failure.," Journal of the Association of Physicians of India, vol. 43, no. 7, pp. 462-463, 1995.

[15] I. M. Murray-Lyon, A. H. Orr, B. Gazzard, J. Kohn, and R. Williams, "Prognostic value of serum alpha fetoprotein in fulminant hepatic failure including patients treated by charcoal haemoperfusion," Gut, vol. 17, no. 8, pp. 576-580, 1976.

[16] A. Varshney, R. Gupta, S. K. Verma, and S. Ahmad, "Alphafetoprotein as a prognostic marker in acute liver failure: a pilot study," Tropical Doctor, vol. 47, no. 3, pp. 202-205, 2017.

[17] H. Ohnishi, J. Sugihara, H. Moriwaki et al., "Acute-on-chronic liver failure," Journal of Hepatology, vol. 13, no. 12, pp. 2128-2139, 1995.

[18] A. Katoonizadeh, W. Laleman, C. Verslype et al., "Early features of acute-on-chronic alcoholic liver failure: A prospective cohort study," Gut, vol. 59, no. 11, pp. 1561-1569, 2010.

[19] S. Lin, J. Chen, M. Wang et al., "Prognostic nomogram for acuteon-chronic hepatitis B liver failure," Oncotarget, vol. 8, no. 65, pp. 109772-109782, 2017.

[20] S. M. Zhu, Y. Waili, X. T. Qi, Y. M. Chen, and Y. F. Lou, "Lymphocyte-monocyte ratio at admission predicts possible outcomes in patients with acute-on-chronic liver failure," European Journal of Gastroenterology \& Hepatology, vol. 29, no. 1, pp. 31-35, 2017. 
[21] B. Chen and S. Lin, "Albumin-bilirubin (ALBI) score at admission predicts possible outcomes in patients with acute-onchronic liver failure," Medicine (United States), vol. 96, no. 24, Article ID e7142, 2017.

[22] M.-H. Zheng, K.-Q. Shi, Y.-C. Fan et al., "A Model to Determine 3-Month Mortality Risk in Patients With Acute-on-Chronic Hepatitis B Liver Failure," Clinical Gastroenterology and Нераtology, vol. 9, no. 4, pp. 351-e3, 2011.

[23] J. Xin, W. Ding, S. Hao et al., "Serum macrophage inflammatory protein $3 \alpha$ levels predict the severity of HBV-related acute-onchronic liver failure," Gut, vol. 65, no. 2, pp. 355-357, 2016.

[24] Y. Yan, L. Mai, Y.-B. Zheng et al., "What MELD score mandates use of entecavir for ACLF-HBV HBeAg-negative patients?" World Journal of Gastroenterology, vol. 18, no. 33, pp. 46044609, 2012.

[25] T. Wu, J. Li, L. Shao et al., "Development of diagnostic criteria and a prognostic score for hepatitis B virus-related acute-onchronic liver failure," Gut, p. gutjnl-2017-314641.

[26] L. Chen, Y. Lou, Y. Chen, and J. Yang, "Prognostic value of the neutrophil-to-lymphocyte ratio in patients with acute-onchronic liver failure," International Journal of Clinical Practice, vol. 68, no. 8, pp. 1034-1040, 2014.

[27] L. E. Schmidt and K. Dalhoff, "Alpha-fetoprotein is a predictor of outcome in acetaminophen-induced liver injury," Hepatology, vol. 41, no. 1, pp. 26-31, 2005.

[28] F. V. Schiodt, G. Ostapowicz, N. Murray et al., "Alpha-fetoprotein and prognosis in acute liver failure," Liver Transplantation, vol. 12, no. 12, pp. 1776-1781, 2006.

[29] G. I. Abelev, S. D. Perova, N. I. Khramkova, Z. A. Postnikova, and I. S. Irlin, "Production of embryonal $\alpha$-globulin by transplantable mouse hepatomas," Transplantation, vol. 1, no. 2, pp. 174-180, 1963.

[30] Y. R. Krishna, V. A. Saraswat, K. Das et al., "Clinical features and predictors of outcome in acute hepatitis A and hepatitis E virus hepatitis on cirrhosis," Liver International, vol. 29, no. 3, pp. 392-398, 2009.

[31] H. Garg, A. Kumar, V. Garg, P. Sharma, B. C. Sharma, and S. K. Sarin, "Clinical profile and predictors of mortality in patients of acute-on-chronic liver failure," Digestive and Liver Disease, vol. 44, no. 2, pp. 166-171, 2012. 


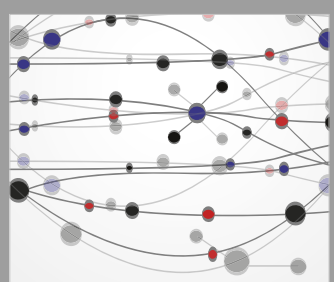

The Scientific World Journal
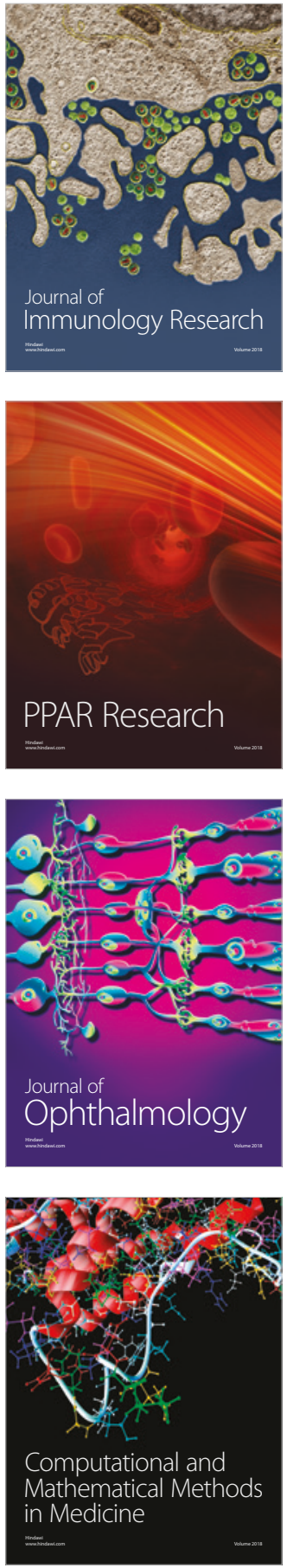

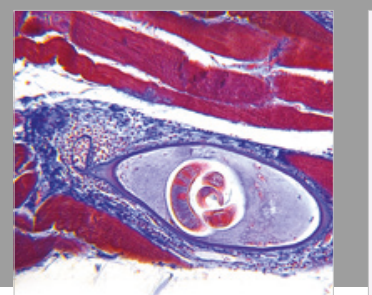

Gastroenterology Research and Practice

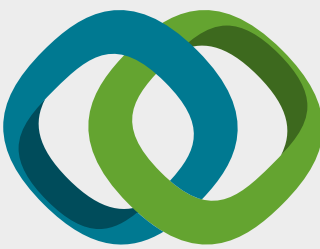

\section{Hindawi}

Submit your manuscripts at

www.hindawi.com
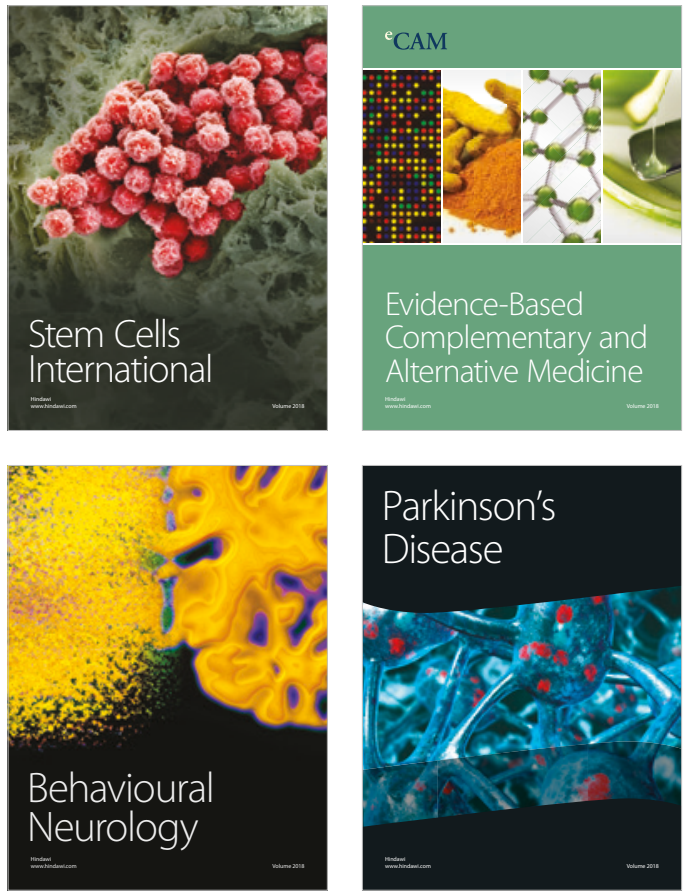

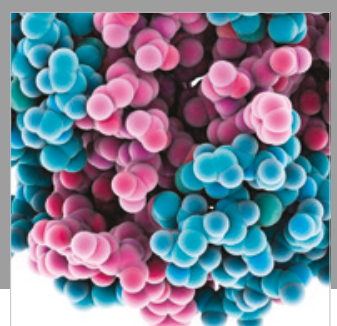

ournal of

Diabetes Research

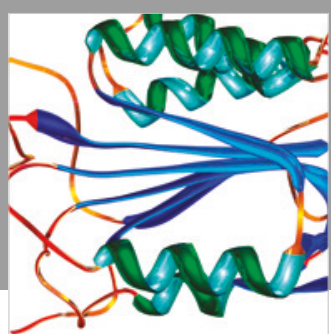

Disease Markers
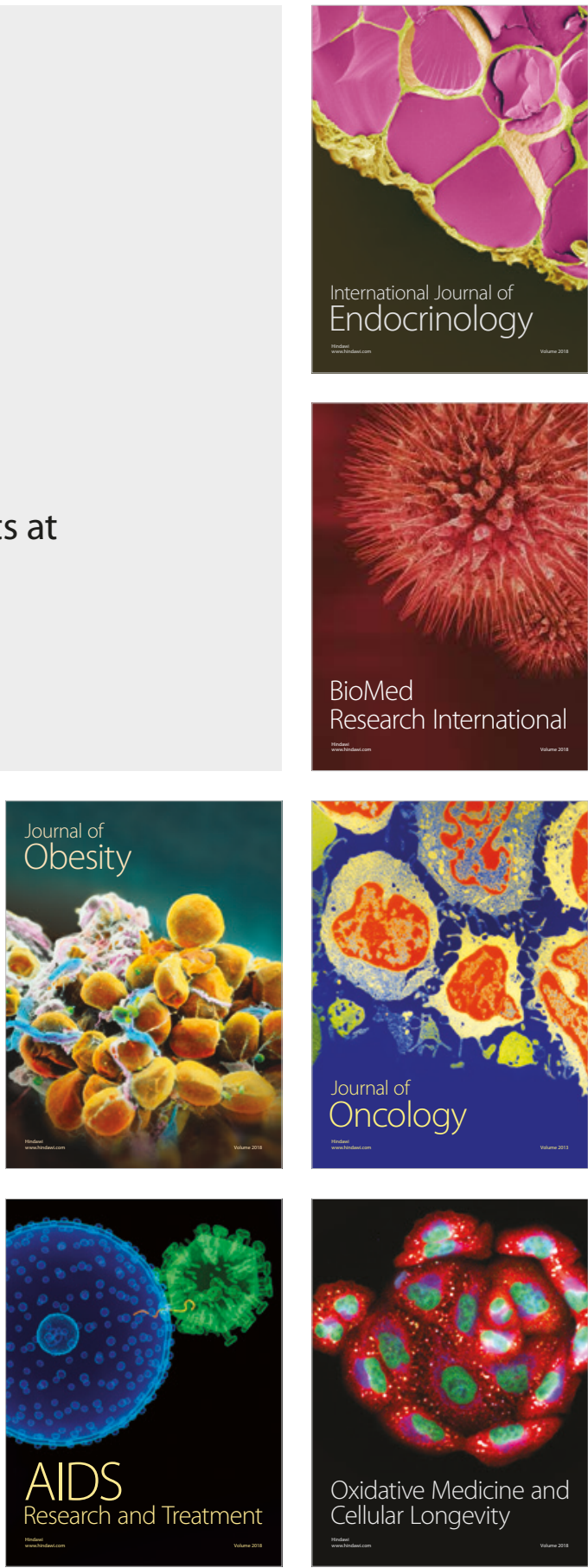